\title{
A preliminary evaluation of the Edinburgh dicentric hunter
}

\author{
PAUL FINNON AND DAVID LLOYD
}

National Radiological Protection Board, Chilton, Didcot, Oxon, OX11 ORQ, UK

\begin{abstract}
Dicentric/automatic scoring/lymphocyte
Computer programs have recently been developed to enable automatic detection of dicentric chromosomes on microscope slides. Theoretical calculations based on the probable limits of resolution of such a system suggested that about two thirds of dicentrics might be detectable. We present here some preliminary results from an ongoing evaluation of an automatic dicentric hunter using slides of lymphocyte preparations which have also been examined by eye. The data indicate an efficiency approaching $40 \%$ in dicentric detection.
\end{abstract}

\section{INTRODUCTION}

Biological dosimetry was established in the 1960 s and has proved very useful in radiological protection for confirming accidental overexposures to radiation and assessing the dose. For a number of reasons ${ }^{1)}$ the analysis of metaphases from peripheral blood lymphocytes for the presence of dicentric aberrations is the technique of choice. Other types of unstable aberrations; centric rings and acentric fragments are also observed but are rarely used in quantifying the dose. A drawback of biological dosimetry is that compared with, for example, processing a film badge, it is much more expensive as it is labour intensive. Because dicentrics are non-constitutional anomalies which may often be present in only a small proportion of the cells, it is usually necessary to examine a large number of metaphases. To produce an estimate of dose below $1 \mathrm{~Gy}$ that carries sufficiently small statistical uncertainty to be useful, at least 500 metaphases should be scored.

Automation of analysis has already made a significant impact in that there are now several fast and accurate metaphase finders commercially available. These can present cells ranked for the quality of spread and staining, in focus and at high magnification, with few false positives or negatives. The technician then examines the metaphases through the microscope counting the number of objects in the spread, and recording any aberrations. The next logical step in automation would be to replace or reduce the need for visual examination. In view of the key importance of the dicentric, this is the aberration that has been targeted for automatic detection. 


\section{METHOD}

The Cytoscan metaphase finder that has been operating in the NRPB laboratory since 1984 has recently been upgraded. This has enabled the system to use the "dicentric hunter" programs that have recently been developed by Piper and colleagues at the Medical Research Council Human Genetics Unit in Edinburgh, UK. These procedures have been described elsewhere ${ }^{2-4)}$.

In brief, Giemsa-stained metaphases are located by the standard metaphase finding procedure that is supplied with all Cytoscan instruments. This has been described and evaluated previously ${ }^{5}$. The metaphases are then recalled under $\times 1000$ magnification and the image of each is digitised and stored on a Winchester disc. Each metaphase image is then examined automatically to count the number of objects and touching or overlapping objects are identified and automatically separated. Algorithms for detecting centromeres, and hence dicentrics, are then applied. These analyse the chromosome boundary and also the density distribution along the chromosome axis. A second screening procedure is then applied to attempt to detect false positives. These are due to chromosomes where the chromatids cross over or composite objects where automatic segregation has failed to separate touching or overlapping chromosomes. Information on those metaphases containing a candidate dicentric are then transferred to a "positives" file for later review. Up to this point the procedure does not require the presence of a technician and therefore can run overnight.

On completion of this stage the positives file is reviewed by a technician. Candidate dicentrics are displayed on a TV monitor and the metaphase is also brought into view in the microscope. The technician then has the choice of confirming or rejecting the dicentric from the screen or from the microscope. In practice we reject obvious false positives simply from the TV screen and all other objects are examined in the microscope. The true dicentrics are then collated into a "results" file and score sheets can be printed. In our laboratory the results are arranged so that it is possible to derive the location of each metaphase assessed, the number of objects that it contained and the number of candidate and confirmed dicentrics (or dicentric equivalents, ie. tri- and quadracentrics).

\section{EFFICIENCY OF DICENTRIC DETECTION}

\section{Theoretical}

Because of the variability of appearance of dicentrics, no automatic system can be guaranteed to find them with $100 \%$ efficiency. The false negative rate is considerably improved if, from the outset, only those candidate aberrations that conform to a certain strict definition are considered, and this will obviously include the more easily identified dicentrics. For example, dicentrics tend to be larger objects than the medium sized chromosomes and so a size limit may be imposed. Identification of dicentrics is more certain if they are "metacentric" ie. both centromeres are located away from the tips of the aberrant chromosome. The centro- 
meres in a "metacentric" dicentric should also be separated by a certain distance. Exclusion of those candidate objects not conforming to such criteria will obviously result in a number of true dicentrics being ignored but will lead to far fewer ambiguous images and false positives being referred to the operator.

Prior to incorporating a dicentric hunting capacity into our instrument, a theoretical exercise was carried out to predict the numbers of false negatives (ie. missed dicentrics) that would arise due to the selection criteria. Pairs of breaks were randomly generated in the male and female human karyotype. Misrepair was assumed with recombination to form unstable type aberrations. For simplicity the probability of a break occurring in a $G_{0}$ arm was assumed to be proportional to its length as observed at metaphase ${ }^{6)}$. Therefore, no account was taken of "hot spots" or areas of preferred exchange. The criteria for deciding whether a dicentric would be detected due to its being acrocentric, centromeres too close together or overall length too short were determined after consultation with Dr. Piper. These are as follows:

a) The dicentric was rejected if the distance between either end and a centromere was less than $0.36 \%$ of the total length of all 92 chromosome arms. This was chosen because it includes the short arms of the normally acrocentric chromosomes (nos. 13-15, 21, 22 and Y) but excludes the short arm of no.18 which is the shortest of all other arms.

b) The dicentric was rejected if the distance between centromeres was less than $0.6 \%$ of the total length of the 92 arms.

c) The overall length of the dicentric should be equal to or greater than the length of normal chromosome no. 12.

In Table 1 is shown the results of 5000 pairs of breaks selected using a random number generator. Here, dicentrics are sub-divided into those that would be rejected due to being singly or doubly acrocentric, being metacentric but with centromeres too close or solely being too short. No dicentric is listed more than once because it was rejected on more than one criterion. The equality of numbers of dicentrics generated in each sex is not a chance event; precisely the same set of random numbers was used in each. The sex difference in the rejected dicentrics is due to the involvement of the $\mathrm{Y}$ chromosome in exchanges.

Table 1. Results of a theoretical exercise to predict the numbers of dicentrics that might be detected automatically when 5,000 pairs of breaks are induced at random in the human karyotype.

\begin{tabular}{|c|c|c|c|c|c|c|}
\hline & \multirow{2}{*}{$\begin{array}{c}\text { Total } \\
\text { dicentrics }\end{array}$} & \multirow{2}{*}{$\begin{array}{l}\text { Accepted } \\
\text { dicentrics }\end{array}$} & \multicolumn{4}{|c|}{ Rejected dicentrics } \\
\hline & & & $\begin{array}{l}\text { singly } \\
\text { acrocentric }\end{array}$ & $\begin{array}{c}\text { doubly } \\
\text { acrocentric }\end{array}$ & $\begin{array}{c}\text { close } \\
\text { centromeres }\end{array}$ & too short \\
\hline Male & 4864 & 3163 & 1017 & 75 & 530 & 79 \\
\hline Female & 4864 & 3206 & 969 & 55 & 575 & 59 \\
\hline Mean & 4864 & $3185(65 \%)$ & $993(20 \%)$ & $65(1 \%)$ & $552(11 \%)$ & $69(1 \%)$ \\
\hline
\end{tabular}

In all, 35\% of dicentrics were rejected in this theoretical exercise. This means that using these, probably oversimplified criteria, machine identification of dicentrics might result in a loss of about one third of the available information. Therefore, approximately $50 \%$ more cells 
would need to be examined to generate a dose estimate carrying the same statistical reliability as that based on conventional visual scoring.

\section{Practical}

A comparison is in progress of visual and machine scoring of dicentrics on a number of Giemsa stained slides. Some preliminary results have been extracted for presentation here for a) one slide with a relatively low dicentric yield from an accidentally irradiated subject, b) six replicate slides from an in vitro experiment where a mixture was made of $10 \%$ unirradiated blood and $90 \%$ irradiated to $8 \mathrm{~Gy}$ of $\mathrm{x}$-rays. The data from these six slides have been pooled for presentation.

For both visual and machine scoring, the cells were located by automatic metaphase finding. The listed cells were screened by eye at $\times 200$ magnification and classified as usable or not usable metaphases or débris. At this magnification a technician can reliably identify those cells where the appearance of the spread is adequate for analysis but of course cannot be certain that the spread contains 46 centromeres. For the visual scoring only the usable quality metaphases were examined and any spreads containing 36 or more chromosomes were scored as this approximates to the lower cut-off level of the object count in the dicentric hunting program. For the machine scoring however all listed images irrespective of quality and including débris were digitised and passed to the dicentric hunting procedure. The same operator performed both the visual scoring and the review of machine scored candidates.

Table 2 shows the proportion of the machine scored images that were in each class. Also shown are the number of candidate dicentrics that the instrument presented to the operator and the number judged to be true. This analysis was made in order to determine whether a visual pre-screening, prior to machine scoring, is necessary. There is a possibility that the instrument could attempt to judge chromsomes in cells that are really unsuitable for analysis. This would tend to put up the false positive rate. In addition there is the possibility of bias if dicentrics are likely to occur more frequently in spreads of lesser quality. This is a subjective opinion sometimes made by scorers although Finnon et $\mathrm{al}^{5)}$ have presented data suggesting that this was not the case. It is apparent from the data in Table 2 that the instrument is effective in identifying images that would be judged by eye to be of scorable quality. The final number of true dicentrics that are derived from images that would have been deemed unsuitable for

Table 2. The proportion of images that were judged by eye to be usable or unusable metaphases or débris on the test slides and the distribution of candidate (CD) and true (TD) aberrations recorded by the dicentric hunter.

\begin{tabular}{|c|c|c|c|c|c|}
\hline & Total No. & Images & Usable & Unusable & Débris \\
\hline Low Dose & $\begin{array}{rr} & \text { CD } \\
1104 & \\
& \text { TD }\end{array}$ & $\begin{array}{r}343 \\
44\end{array}$ & $\begin{array}{rrr} & \text { CD } 329 \\
1048 & & \\
& \text { TD } & 43\end{array}$ & $\begin{array}{lll} & \text { CD } 19 \\
56 & & \\
& \text { TD } & 1\end{array}$ & 0 \\
\hline High Dose & $\begin{array}{rr} & \text { CD } \\
1133 & \\
& \text { TD }\end{array}$ & $\begin{array}{l}512 \\
286\end{array}$ & $\begin{array}{rr} & \text { CD } 466 \\
1038 & \\
& \text { TD } 259\end{array}$ & $\begin{array}{ll} & \text { CD } 46 \\
& \text { TD } 27\end{array}$ & $\begin{array}{l}\mathrm{CD} 0 \\
\mathrm{TD} 0\end{array}$ \\
\hline
\end{tabular}


analysis comprises less than $10 \%$ of the total.

Table 2 also shows that there is a considerable difference between the low and high dose material in the proportion of candidates presented by the dicentric hunter that were judged by the operator to be true dicentrics. Of course, one would expect fewer dicentrics at the lower dose and $13 \%(44 / 348)$ were true, whereas the corresponding value for the high dose was $56 \%$ $(286 / 512)$. The number of false positive per cell should be independent of dose and in Table 2 these may be calculated at 0.27 and 0.2 per cell for low and high doses respectively. This value compares quite well with the experience of the Edinburgh laboratory of one false positive per 5 metaphases (Piper, personal communication). The false positive rate is of course modifiable depending on the selection criteria adopted, although for the present study the default settings were not adjusted. More restrictive criteria will reduce the rate but will incur the penalty of failing to identify true dicentrics (false negatives) and so necessitate a larger sample of metaphases to be examined. Less restrictive criteria will increase the false positive rate and so produce a longer list of objects that will have to be reviewed. Both situations increase the amount of operator time and when a larger data set is available, it is hoped that optimal criteria may be determined.

Table 3. A comparison of true dicentric yields in metaphases of $>36$ objects obtained by visual or machine scoring on the same slides.

\begin{tabular}{|l|c|cc|c|c|c|}
\hline $\begin{array}{l}\text { Scoring } \\
\text { method }\end{array}$ & Dose & $\begin{array}{c}\text { No. of } \\
\text { spreads }\end{array}$ & $\begin{array}{c}\text { No. of } \\
\text { chromosomes }\end{array}$ & $\begin{array}{c}\text { No. of } \\
\text { dicentrics }\end{array}$ & $\begin{array}{c}\text { Dic. per } \\
\text { spread }\end{array}$ & $\begin{array}{c}\text { Dic. per } \\
\text { chromosome } \\
\left(\times 10^{-4}\right)\end{array}$ \\
\hline Visual & Low & 1552 & 71,303 & 170 & 0.11 & 23.8 \\
\hline \multirow{2}{*}{ Machine } & High & 2408 & 110,618 & 2046 & 0.85 & 185.0 \\
\hline & Low & 1104 & 47,620 & 44 & $0.04(36 \%)$ & $9.2(39 \%)$ \\
\hline
\end{tabular}

The main reason for the listing of false positives was probably failure of segmentation, ie. the automatic procedure to separate two or more chromosomes in close proximity. One sub-set of the data, 171 metaphase spreads (7,142 chromosomes) that were present on one of the high dose slides, was used to determine the likely basis for the false positives. Seventy-four candidates were found and 43 were judged to be false. Seventeen were single chromosomes with twisted or overlapping chromatids; 18 were two or more very close but not touching chromosomes; 7 were a pair of overlapping chromosomes ( 5 of which were end to end overlaps) and one was a normal chromosome partially obscured by a piece of débris. Eventually all false positive in this study will be examined and classified to provide data indicating where improvements to segmentation should be sought. It was apparent from the one slide so far examined that the failure to segment correctly usually occurred in metaphases where there was some cytoplasm residue that had taken up Giemsa stain so that the space between chromosomes was not clear. This is a situation which can be improved by use of a more DNA specific stain. 
Also it is possible to remove residual cytoplasm by placing slides for a few minutes in $5 \mathrm{~N}$ hydrochloric acid prior to staining in Giemsa. Eventually the best solution to this problem would be to use a regimen that aims to produce uniform quality preparations well suited to automatic scoring ${ }^{7}$.

It should be pointed out that although all cells were located by automatic metaphase finding, the configuration of the upgraded Cytoscan is such that the old and the new systems each have separate metaphase finding software. For the present it is not possible to score visually and automatically precisely the same list of metaphases. The two sets of software have slightly different default settings and the more recent program searches a smaller area of the slide. Nevertheless a considerable proportion of the metaphases examined were common to both lists. As cell locations are recorded it will be possible to make a precise comparison on a cell to cell basis but at present the data are not easily extractable in a convenient form for this. Therefore in Table 3 we present mean yields from the slides.

It is a convention with visual scoring for biological dosimetry that the yield of dicentrics in "complete" cells is used for comparison with in vitro dose response data. These are cells with 46 centromeres and each dicentric is accompanied by an acentric fragment. The dicentric hunter is however only programmed to identify polycentric chromosomes; not acentrics or centric rings. Thus it is not possible to score automatically using the same criteria as visual analysis. Of course, during the final review of candidates visual examination of the metaphase for accompanying acentrics is possible but this adds to operator time.

For dosimetry purposes it may be equally acceptable simply to consider the yield of all dicentrics irrespective of completeness provided that one controls with fluorescence plus Giemsa staining to ensure that the spreads are more than $95 \%$ first divisions ${ }^{1}$. However, on any slide there is a proportion of metaphases that do not contain 46 centromeres. Usually there are fewer and the main cause of this is the break-up of the clusters of chromosomes when they are dropped onto the glass. The dicentric hunter is programmed to search in incomplete spreads and thus it may be more accurate to consider the yields of dicentrics per chromosome rather than per cell. Information on the inter-cellular distribution of dicentrics must nevertheless be available to enable overdispersion to be detected in cases of non-uniform or part-body irradiation $^{1)}$. In Table 3 the yields are presented in both ways.

In comparing the values in Table 3 of mean yields of true dicentrics per spread, the machine scored yields for the low and high dose material are respectively $36 \%$ and $30 \%$ of those for the visual scoring. These two values are clearly well below the theoretical maximum of $65 \%$ detection efficiency discussed earlier in this paper. They are however close to a value of nearly $40 \%$ that has recently been obtained in a collaborative trial between the Edinburgh and Pittsburgh laboratories ${ }^{3)}$. If the yields are expressed on a per chromosome basis then both rise slightly, but probably not significantly, to $39 \%$ and $32 \%$ respectively. The efficiency of dicentric detection appears to be slightly reduced for the higher dose and this was also observed by Bayley et $\mathrm{al}^{3)}$. In compiling the present data a feature of the programs that may explain this became apparent. One of the procedures to minimise the false positive rate automatically discards those spreads that contain many $(>5)$ candidate dicentrics. This will be rectified, but for the $8 \mathrm{~Gy}$ irradiated material in the present study, it is known from the visual scoring that 
$28 \%(584 / 2,046)$ of the dicentrics were contained in such metaphases. Allowing for this, by multiplying the $30 \%$ and $32 \%$ values in Table 3 by 1.28 brings them close to $40 \%$.

\section{CONCLUSIONS}

1. In spite of the preliminary nature of the current evaluation the performance values obtained were close to those of the Edinburgh-Pittsburg trial ${ }^{3)}$.

2. Automatic metaphase finding followed by visual scoring may usefully incorporate a low magnification visual pre-screening in order to cull the list to remove spreads of insufficient quality for visual scoring. This step does not seen necessary for automatic dicentric hunting.

3. Comparison of the true dicentric yields from visual and machine scoring of the same slides has produced values that are initially disappointing indicating only $35-40 \%$ efficiency in automatic detection of dicentrics with a slightly worse performance at high dose. However, it should be stressed that these are preliminary conclusions and further studies are in progress with slides of greater variety of spread quality in order to determine consistency of performance. It will also be necessary to make a more precise comparison on a cell to cell basis rather than mean dicentric yields per slide.

4. Programs for dicentric hunting are still evolving and a principal objective of this evaluation is to identify software features that can be improved.

\section{REFERENCES}

1. IAEA (1986) Biological dosimetry: chromosomal aberration analysis for dose assessment. Technical Report Series No. 260. International Atomic Energy Agency, Vienna.

2. Piper, J., Towers, S., Gordon, J., Ireland, J. and McDougall, D. (1988) Hypothesis combination and context sensitive classification for chromosome aberration scoring. Pattern Recognition and Artificial Intelligence. Ed. E. S. Gelsema, L. N. Kanal. pp. 449-460, Elsevier, Amsterdam.

3. Bayley, R., Carothers, A., Chen, X., Farrow, S., Gordon, J., Ji, L., Piper, J., Rutovitz, D., Stark, M. and Wald, N. (1991) Radiation dosimetry by automatic image analysis of dicentric chromosomes. Mutation Research 253: 223-235.

4. Piper, J. and Sprey, J. (1992) Adaptive classifiers for dicentric chromosomes. J. Radiat. Res. 33: Suppl. $157-170$.

5. Finnon, P., Lloyd, D. C. and Edwards, A. A. (1986) An assessment of the metaphase finding capability of the Cytoscan. Mutation Research 164: 101-108.

6. Paris Conference (1971) Standardisation in human cytogenetics. Birth defects: Original article series, $8 ; 7$. The National Foundation, New York.

7. Yamada, K., Kakinuma, K., Tateya, H. and Toya, M. (1992) Development of an instrument for chromosome slide preparation. J. Radiat. Res. 33: Suppl. 242-249. 